\title{
Space Gravity Spectroscopy - determination of the Earth's gravitational field by means of Newton interpolated LEO ephemeris Case studies on dynamic (CHAMP Rapid Science Orbit) and kinematic orbits
}

\author{
T. Reubelt, G. Austen, and E. W. Grafarend \\ Geodetic Institute, Stuttgart University, Geschwister-Scholl-Str. 24/D, D-70174 Stuttgart, Germany
}

\begin{abstract}
An algorithm for the (kinematic) orbit analysis of a Low Earth Orbiting (LEO) GPS tracked satellite to determine the spherical harmonic coefficients of the terrestrial gravitational field is presented. A contribution to existing long wavelength gravity field models is expected since the kinematic orbit of a LEO satellite can nowadays be determined with very high accuracy in the range of a few centimeters. To demonstrate the applicability of the proposed method, first results from the analysis of real CHAMP Rapid Science (dynamic) Orbits (RSO) and kinematic orbits are illustrated. In particular, we take advantage of Newton's Law of Motion which balances the acceleration vector and the gradient of the gravitational potential with respect to an Inertial Frame of Reference (IRF). The satellite's acceleration vector is determined by means of the second order functional of Newton's Interpolation Formula from relative satellite ephemeris (baselines) with respect to the IRF. Therefore the satellite ephemeris, which are normally given in a Body fixed Frame of Reference (BRF) have to be transformed into the IRF. Subsequently the Newton interpolated accelerations have to be reduced for disturbing gravitational and non-gravitational accelerations in order to obtain the accelerations caused by the Earth's gravitational field. For a first insight in real data processing these reductions have been neglected. The gradient of the gravitational potential, conventionally expressed in vector-valued spherical harmonics and given in a Body Fixed Frame of Reference, must be transformed from BRF to IRF by means of the polar motion matrix, the precession-nutation matrices and the Greenwich Siderial Time Angle (GAST). The resulting linear system of equations is solved by means of a least squares adjustment in terms of a Gauss-Markov model in order to estimate the spherical harmonics coefficients of the Earth's gravitational field.
\end{abstract}

Key words. space gravity spectroscopy, spherical harmonics series expansion, GPS tracked LEO satellites, kinematic

Correspondence to: T. Reubelt

(reubelt@gis.uni-stuttgart.de) orbit analysis, Newton interpolation

\section{Reference frames}

First, the beforehand mentioned transformation between the Inertial Reference Frame and the Body Fixed Reference Frame is considered for this contribution more an issue of the operational point of view (software development) since the underlying theoretical aspects are well known, eg. McCarthy (1996). The resulting transformation matrix $\mathbf{R}(t)$ contains the parameters of nutation, precession, polar motion and Greenwich siderial time. Corrections for the nutation model and the parameters for polar motion and Greenwich siderial time are delivered by the Bulletins of the International Earth Rotation Service (IERS).

\section{Representation of the Earth's gravitational field - the spherical harmonics series expansion}

For the description of the gravitational field of the Earth we use a spherical harmonics series expansion. Equation (1) defines this series expansion of the gravitational potential $U(\lambda, \varphi, r)$ as an infinite sum. In our approach the summation is only executed to a maximum sensitivity degree $L$, constituted by the measurement principle of CHAMP. $G M$ denotes the geocentric gravitational constant, $R$ the mean radius of the Earth, $l$ and $m$ degree and order of the spherical harmonics series expansion and $P_{l, m}^{*}(\sin \varphi)$ are Ferrer's fully normalized associated Legendre functions. $u_{l, m}$ identify the unknown spherical harmonic coefficients which we aim to determine. These coefficients $u_{l, m}$ can be divided into coefficients $c_{l, m}$ (cosine term, $m \geq 0$ ) and $s_{l, m}$ (sine term, $m<0)$.

Ferrer's fully normalized associated Legendre-functions $P_{l, m}^{*}(\sin \varphi)$ are obtained from the associated Legendrefunctions $P_{l, m}(\sin \varphi)$ by normalisation according to Eq. (2). For an efficient and numerical stable computation of the as- 


\section{Table 1}

spherical harmonics series expansion of the Earth's gravity field

$$
\mathrm{U}(\lambda, \varphi, r)=\frac{\mathrm{GM}}{\mathrm{R}} \lim _{L \rightarrow \infty} \sum_{l=0}^{L} \sum_{m=-l}^{+l}\left(\frac{\mathrm{R}}{r}\right)^{l+1} u_{l, m} P_{l,|m|}^{*}(\sin \varphi)\left\{\begin{array}{cc}
\cos (m \lambda) & m \geq 0 \\
\sin (|m| \lambda) & m<0
\end{array}\right.
$$

"Ferrer's fully normalized associated Legendre-functions"

$$
P_{l, m}^{*}(\sin \varphi):=\left\{\begin{array}{cc}
\sqrt{2(2 l+1) \frac{(l-|m|) !}{(l+|m|) !}} \cdot P_{l, m}(\sin \varphi) & ; m \neq 0 \\
\sqrt{2 l+1} \cdot P_{l, 0}(\sin \varphi) & ; m=0
\end{array}\right.
$$

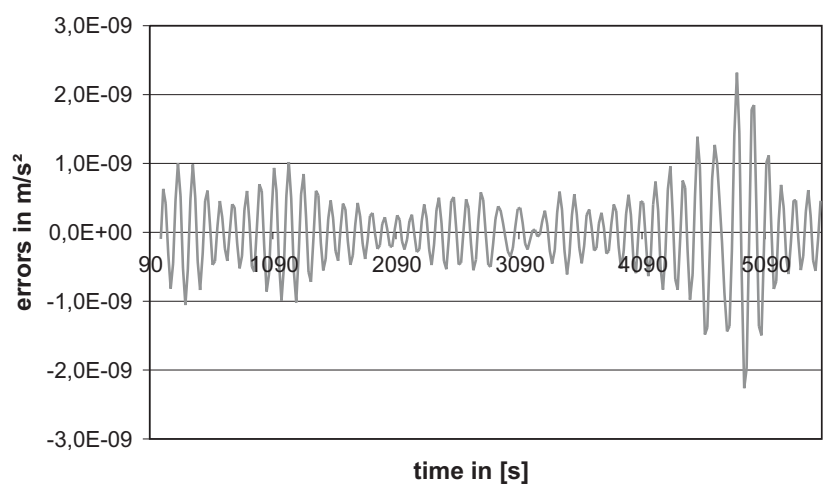

Fig. 1. Interpolation errors of Newton interpolated accelerations $\boldsymbol{X}$ for one simulated CHAMP revolution based on EGM96 up to degree/order 50/50; sampling time $\Delta t=30 \mathrm{~s}$; 9-point scheme; $x$ axis: time in [s]; $y$-axis: acceleration error in $\left[\mathrm{m} / \mathrm{s}^{2}\right]$

sociated Legendre functions recurrence formulae are applied (Koop and Stelpstra, 1989; Belikov and Taybatarov, 1992).

\section{Determination of accelerations by means of Newton interpolation}

For the determination of accelerations from GPS tracked absolute ephemeris $\boldsymbol{X}(t)$ or baselines $\Delta \boldsymbol{X}(t)$ we have chosen Newton's interpolation formula for equidistant sampling points (Engeln-Müllges and Reutter, 1966). Based on the zero order functional, Eq. (3), we derive Newton's second order interpolation formula in Eq. (7) which is computed by a product-sum of forward differences (Eq. 5) and the second order functional of Newton's base polynomials (Eq. 8). Newton's base polynomials (Eq. 6) contain the time difference quotient $q$ (Eq. 4) and are received via $q$ over $i$. The forward differences are originally determined from absolute ephemeris (Eq. 5), but in our case the forward differences can be expressed in terms of relative ephemeris (baselines of adjacent positions, Eq. 9). Since adjacent ephemeris are highly correlated (and thus relative ephemeris can be determined a lot more precise than absolute ones), an improvement of accuracy is realized.

For our purpose, the application of the 9-point scheme has turned out to provide the best approximation (Austen and Reubelt, 2000), and so the following computations and figures are based upon the 9-point interpolation scheme.

In general a $n$-point interpolation scheme can be considered as a mask, which allows the computation of acceleration vectors from sets of $n$ satellite's CoM (Center of Mass) position vectors. This mask is moved successively through the position time series generating an acceleration time series, which has to be further corrected for disturbing accelerations caused by atmospheric drag, solar radiation pressure, third body effects, etc. Again the operational implementation is very laborious, for details one can consult Hartmann and Wenzel (1995), King-Hele (1987) and Wahr (1995). Due to the immense number of observations it is possible to proceed the mask in a non - overlapping way to avoid correlation for consecutive mask positions.

\section{Performance analysis of Newton interpolation}

\subsection{Approximation error}

To examine the quality of the proposed procedure to determine the accelerations of the satellite, we investigated first the approximation behaviour of Newton interpolation for simulated orbit data, neglecting measurement errors. Figure 1 illustrates the approximation behaviour for one simulated CHAMP revolution based upon a degree and order 50/50 EGM96 gravity field (Lemoine et al., 1998). For the whole revolution, the approximation error lies within an accuracy of $3 \cdot 10^{-9} \mathrm{~m} / \mathrm{s}^{2}(0.0003 \mathrm{mGal})$ which is well in the level of the accuracy of the other instruments, for instance the accelerometer.

\subsection{Influence of GPS measurement errors}

In the next step, we analysed the influence of GPSmeasurement errors in the Newton-interpolated accelera- 
Table 2

"principle of Newton interpolation;

from the zero order functional to the second order functional"

zero order functional:

$$
\begin{aligned}
& \mathbf{x}(t)=\mathbf{x}\left(t_{1}\right)+\left(\begin{array}{c}
q \\
1
\end{array}\right) \boldsymbol{\Delta}_{3 / 2}^{1}+\left(\begin{array}{c}
q \\
2
\end{array}\right) \boldsymbol{\Delta}_{2}^{2}+\left(\begin{array}{c}
q \\
3
\end{array}\right) \boldsymbol{\Delta}_{5 / 2}^{3}+\ldots+\left(\begin{array}{c}
q \\
n-1
\end{array}\right) \boldsymbol{\Delta}_{1+(n-1) / 2}^{n-1}=\mathbf{x}\left(t_{1}\right)+\sum_{i=1}^{n-1}\left(\begin{array}{c}
q \\
i
\end{array}\right) \boldsymbol{\Delta}_{1+i / 2}^{i} \\
& \text { subject to } \\
& \text { time difference quotient } \\
& q:=\frac{\left(t-t_{1}\right)}{\left(t_{2}-t_{1}\right)}=\frac{\left(t-t_{1}\right)}{\Delta t} \\
& \Delta_{1}^{0}=\mathbf{x}_{1} \\
& \boldsymbol{\Delta}_{3 / 2}^{1}=\mathbf{x}_{2}-\mathbf{x}_{1} \\
& \Delta_{2}^{2}=\mathbf{x}_{3}-2 \mathbf{x}_{2}+\mathbf{x}_{1} \\
& \boldsymbol{\Delta}_{1+i / 2}^{i}=\sum_{k=0}^{i}(-1)^{i+k}\left(\begin{array}{l}
i \\
k
\end{array}\right) \mathbf{x}_{k+1} \\
& \left(\begin{array}{c}
q \\
1
\end{array}\right)=\frac{q !}{1 !(q-1) !}=q \\
& \left(\begin{array}{l}
q \\
2
\end{array}\right)=\frac{q !}{2 !(q-2) !}=\frac{q^{2}-q}{2} \\
& \left(\begin{array}{c}
q \\
i
\end{array}\right)=\frac{q(q-1)(q-2) \cdots(q-i+1)}{i !}
\end{aligned}
$$

second order functional:

$$
\ddot{\mathbf{x}}(t)=\left(\begin{array}{c}
q \\
1
\end{array}\right)^{\prime \prime} \boldsymbol{\Delta}_{3 / 2}^{1}+\left(\begin{array}{c}
q \\
2
\end{array}\right)^{\prime \prime} \boldsymbol{\Delta}_{2}^{2}+\left(\begin{array}{c}
q \\
3
\end{array}\right)^{\prime \prime} \boldsymbol{\Delta}_{5 / 2}^{3}+\cdots+\left(\begin{array}{c}
q \\
n-1
\end{array}\right)^{\prime \prime} \boldsymbol{\Delta}_{1+(n-1) / 2}^{n-1}=\sum_{i=1}^{n-1}\left(\begin{array}{c}
q \\
i
\end{array}\right)^{\prime \prime} \boldsymbol{\Delta}_{1+i / 2}^{i}
$$
subject to

second order time derivative forward differences in terms of baselines of the binomial coefficients

$$
\begin{aligned}
& \left(\begin{array}{c}
q \\
1
\end{array}\right)^{\prime \prime}=0 \\
& \boldsymbol{\Delta}_{3 / 2}^{1}=\Delta \mathbf{x}_{2,1} \\
& \left(\begin{array}{l}
q \\
2
\end{array}\right)^{\prime \prime}=\frac{1}{\left(t_{2}-t_{1}\right)^{2}} \\
& \left(\begin{array}{c}
q \\
3
\end{array}\right)^{\prime \prime}=\frac{1}{\left(t_{2}-t_{1}\right)^{2}} \cdot(q-1) \\
& \left(\begin{array}{c}
q \\
n-1
\end{array}\right)^{\prime \prime}=\frac{1}{(n-1) !} \sum_{j=0}^{n-2} \frac{\sum_{k=0}^{n-2}\left(\frac{q-j}{q-k}\right)-1}{(q-j)^{2}} \prod_{l=0}^{n-2}(q-l) \\
& \Delta \mathbf{x}_{k+1, k}:=\mathbf{x}_{k+1}-\mathbf{x}_{k}
\end{aligned}
$$

tions. In order to set up an adequate error-simulation function for absolute ephemeris that considers high correlations between adjacent ephemeris, a Gauss-Markov process has been introduced. This process, successfully used by Grafarend and Vanicek (1980) in the weight estimation in levelling and applied to our topic by Austen et al. (2002) has the following properties of the simulated errors of absolute coordinates $e_{i}$ : standard deviation $\sigma\left(e_{i}\right)=10 \mathrm{~cm}$, correla- tion $\rho=0.99$. The simulated errors $e_{n+1, n}$ of baselines are received as differences of position errors $e_{i}$. The parameters of this noise process were estimated from a comparison of real dynamic and kinematic CHAMP orbits, as explained in Sect. 6.

Figures 2a and $\mathrm{b}$ illustrate some results: While the errors of baselines lie within $5 \mathrm{~cm}$, the errors of absolute ephemeris mount up to $15 \mathrm{~cm}$. Thus, the application of baselines in 


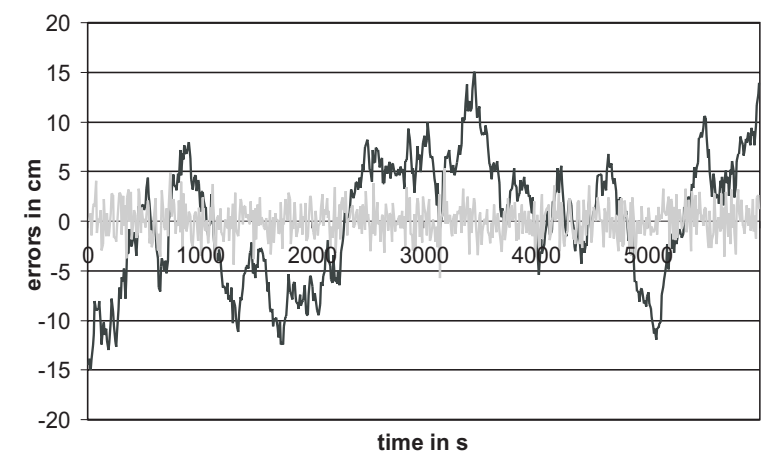

a)

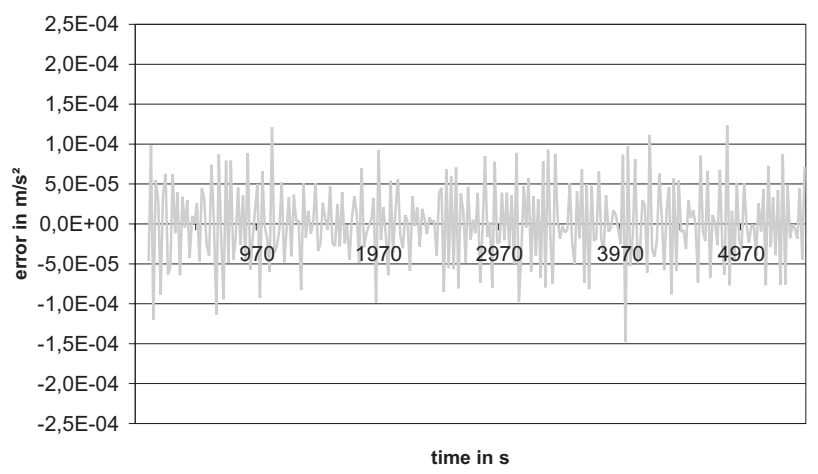

b)

Fig. 2. (a) simulated errors of the tracked coordinates (dark) and baselines (grey) in $\mathrm{cm}$ and (b) errors of Newton interpolated accelerations in $\mathrm{m} / \mathrm{s}^{2}$ (9-point scheme; $\Delta t=30 \mathrm{~s}$ ); standard deviation $\sigma_{x}=10 \mathrm{~cm}$ and correlation $\rho=0.99^{3}=0.970299$ of absolute ephemeris; one revolution of the CHAMP-satellite.

Newton interpolation will improve the accuracy. The errors of the Newton interpolated accelerations obtained from a $30 \mathrm{~s}$ sampling time lie within an accuracy of a few mGal. Due to the immense amount of observations from a 5 years mission duration of CHAMP this accuracy is sufficient to estimate a long wavelength geoid with an accuracy of at least one dm, as estimated from simulations (Reubelt et al., 2002). More details on the derivation of the vector-valued second order functional of Newton's interpolation formula can be found in Austen and Reubelt (2000) and Austen et al. (2002).

\section{System of equations}

After the determination of the satellite's acceleration vector by means of Newton's interpolation formula we have to compute the gradient of the gravitational Potential in the IRF. Therefore the partial derivatives of the gravitational potential $U(\lambda, \phi, r)$, Eq. (1), w.r.t. the spherical coordinates $(\lambda, \phi, r)$ have to be determined, which are transformed in partial derivatives w.r.t. Cartesian BRF coordinates by means of the chain rule. Subsequently this Cartesian gradient is rotated into the IRF by means of the rotation matrix $\mathbf{R}^{T}$, where it is balanced by Newton's law of motion with the satellite's

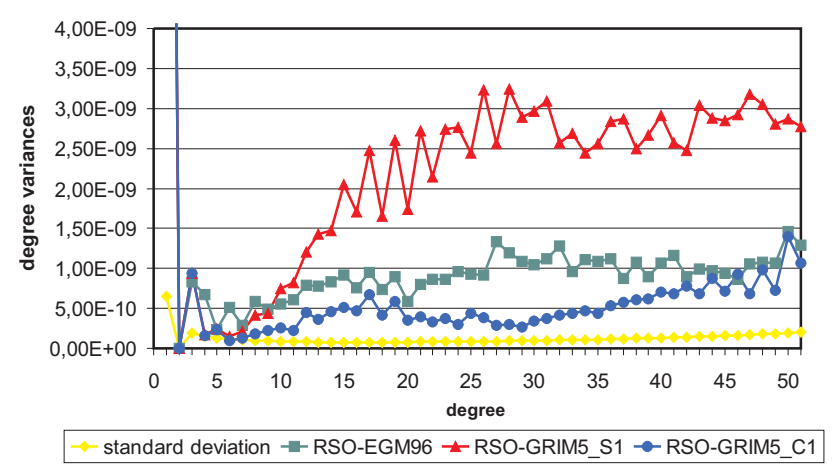

Fig. 3. Degree variances of the estimated standard deviation and the differences between the recovered coefficients and various existing gravity models.

acceleration vector. The resulting linear system of equations is solved for the spherical harmonic coefficients by applying a least squares adjustment in terms of a Gauss-Markov model.

\section{Results}

In this section we provide some results that have been obtained from an analysis of preliminary real CHAMP orbit data sets. The model error as well as accuracy estimates from error simulations of our method have already been tested in previous papers by Austen and Reubelt (2000), Austen et al. (2002) and Reubelt et al. (2002). There, a RMS of the geoid for an error-free simulation of a 1-week-arc (modelerror) in the sub-mm range for degree/order 30/30 was obtained, which is sufficient for CHAMP-data analysis. From an error simulation study with the variance of coordinates $\sigma_{X}=10 \mathrm{~cm}$ and the correlation of $\rho=0.99$, which seems to be a realistic for kinematic orbits according to Reubelt et al. (2002) a $R M S$ of the geoid of $23 \mathrm{~cm}$ was received. Regarding the complete CHAMP-mission, a geoid accuracy of below $10 \mathrm{~cm}$ seems to be possible, which is at least a confirmation of present geoid models. An improving quality of kinematic orbits gives rise to hope for a higher geoid accuracy (Svehla and Rothacher, 2002b). In order to obtain a first insight in real data processing with the proposed method, we have analysed two short preliminary CHAMP orbit data sets in this section.

First we have analysed the CHAMP RSO (Michalak et al., 2002) in the period of 1 July - 16 August 2001, which is sampled in the interval of $\Delta t=30 \mathrm{~s}$. The CHAMP RSO is a dynamic orbit based on a taylored GRIM5-C1 (Gruber et al., 2000) model, thus the determined accelerations of the RSO will be highly correlated to GRIM5-C1. The estimated gravity field model should be comparable to GRIM5_C1, and therefore we have made comparisons to this model. Due to a good condition number of the normal matrix of about 700 , no regularisation had been applied. For a first case study the Newton interpolated accelerations were not reduced from 


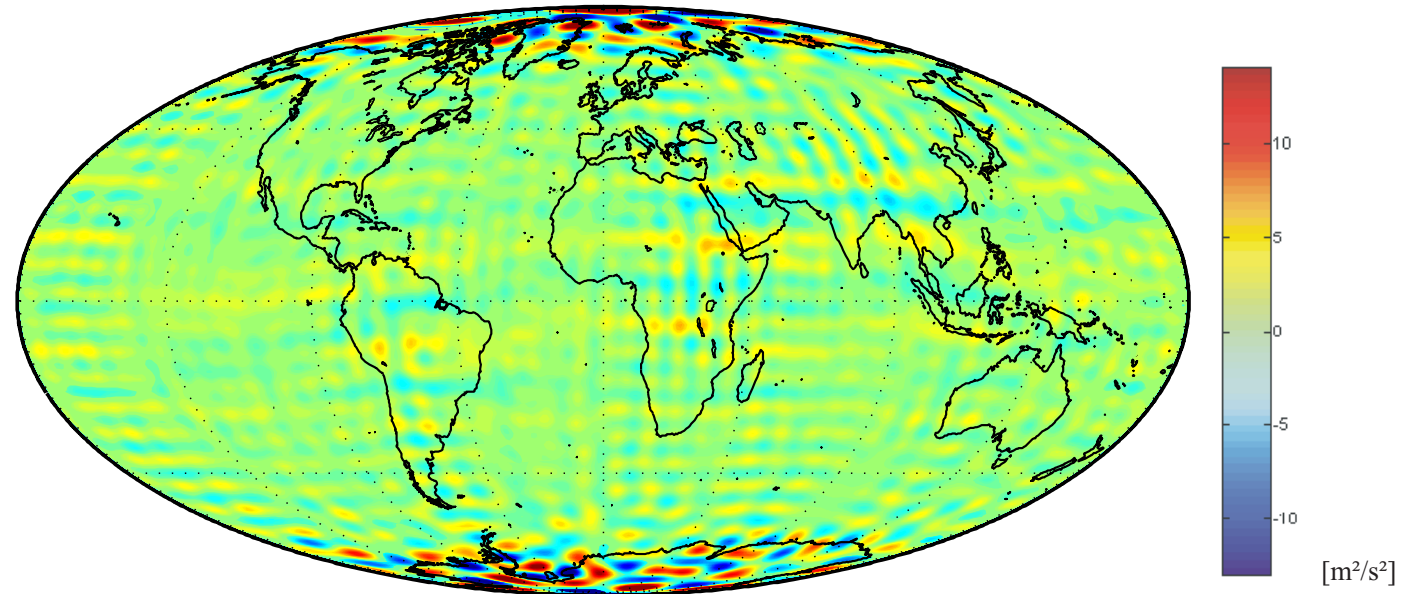

Fig. 4. Difference between recovered geopotential from a 45-days CHAMP-RSO and GRIM5_C1 up to degree/order 50/50 on the surface of a reference Earth with $R=6371 \mathrm{~km}$; without $c_{20}$ term; $R M S_{P o t}=2.8 \mathrm{~m}^{2} / \mathrm{s}^{2}$

disturbing gravitational accelerations (third body attraction of sun, moon, other planets, ocean tides, solid Earth tides ...). Their effect on the geopotential is considered to be in the size of $1-2 \mathrm{~m}^{2} / \mathrm{s}^{2} \cong \hat{\sigma}_{\text {geoid }}=10 \mathrm{~cm}$ (in general) with maximum values of $10 \mathrm{~m}^{2} / \mathrm{s}^{2} \cong \hat{\sigma}_{\text {geoid }}=1 \mathrm{~m}$. Furthermore, the accelerometer measurements are omitted, since they still contain large biases. After publication of these biases by GFZ (GeoForschungsZentrum Potsdam), the reduction of non conservative disturbing accelerations measured by the accelerometer will be carried out. Another possibility, which is in discussion, is the estimation of the accelerometer biases together with the spherical harmonic coefficients in one adjustment. The effect of negligence of the accelerometer measurements is about the same as the influence of the disturbing gravitational forces.

Figure 4 illustrates the differences between the recovered geopotential from a 45-days CHAMP RSO and from GRIM5_C1 up to degree and order 50/50 on the surface of a reference sphere with $R=6371 \mathrm{~km}$. The root mean square $(R M S)$ of the difference is about $2.8 \mathrm{~m}^{2} / \mathrm{s}^{2}$, which corresponds to a $R M S$ of geoidal undulations of $28 \mathrm{~cm}$, while the $R M S$ of the difference of a recovery up to degree/order $30 / 30$ is $1.4 \mathrm{~m}^{2} / \mathrm{s}^{2}$ (as expected due to a loss of signal strength, caused by the term $\left.(R / r)^{l}\right)$ for higher degrees $l$ at satellite altitude). This error is, as visible in the Fig. 4, mainly caused by the polar data gap due to the inclination of the CHAMP orbit. At the non-polar regions, the difference to GRIM5_C1 is smoother and mostly smaller than $1-2 \mathrm{~m}^{2} / \mathrm{s}^{2}$ with the highest errors in the mountain areas, for instance the Himalayas or the Andes, where the gravity field is rough. Small oscillations may be caused by the aliasing effect, since the gravity field was solved only to a maximum degree $L$.

To exhibit the negative effect of signal loss and downward continuation, the differences to the GRIM5_C1 model up to degree/order 50/50 have also been computed at satellite altitude $(h=400 \mathrm{~km})$. The recovered geopotential at satellite altitude is smoother $\left(R M S_{P o t}=1.1 \mathrm{~m}^{2} / \mathrm{s}^{2}\right)$ than the computed geopotential on the reference sphere. This is the effect of the signal damping at satellite altitude by $(R / r)^{l}$, thus the higher frequency parts can not be discovered by satellite measurements at least not by CHAMP, as accurate as by terrestrial measurements. Furthermore a well known fact is clarified: While the differences are small at satellite altitude, they grow very fast with decreasing altitude due to an enhancement of noise by downward continuation. The differences on the surface are 4.5 times higher than at satellite altitude.

Figure 3 presents the error degree-variances to various models (EGM96, GRIM5_C1 and GRIM5_S1; Biancale et al., 2000), as well as the estimated standard deviations of our computations. It shows that the recovered potential fits best to the GRIM5_C1 model. Especially the differences to the satellite-only model GRIM5_S1 are very high. That can be explained by lower accuracy and sensitivity of the former included satellite data as opposed to CHAMP data. The standard deviation of the coefficients, which is estimated from the Gauss-Markov model is smaller than the differences to the previous models, which may be caused also by the errors of these previous models. Probably the errors of the estimated coefficients are higher than the standard deviation, which is very small due to the (smooth) dynamic determination of the RSO.

Subsequently we have analysed kinematic orbits, which are more erroneous, but not based on a force model in terms of a gravity field, and thus will lead to an independent gravity field solution. For our investigations we took a real preliminary kinematic CHAMP orbit of a 11-days time period, 20 to 30 May 2001, sampled at $\Delta t=30 \mathrm{~s}$, which was processed by means of zero difference carrier phase measurements by Svehla and Rothacher (2002a). In order to estimate the accuracy of the kinematic orbit as well as the accuracy of the estimated baselines and accelerations, we made a comparison 


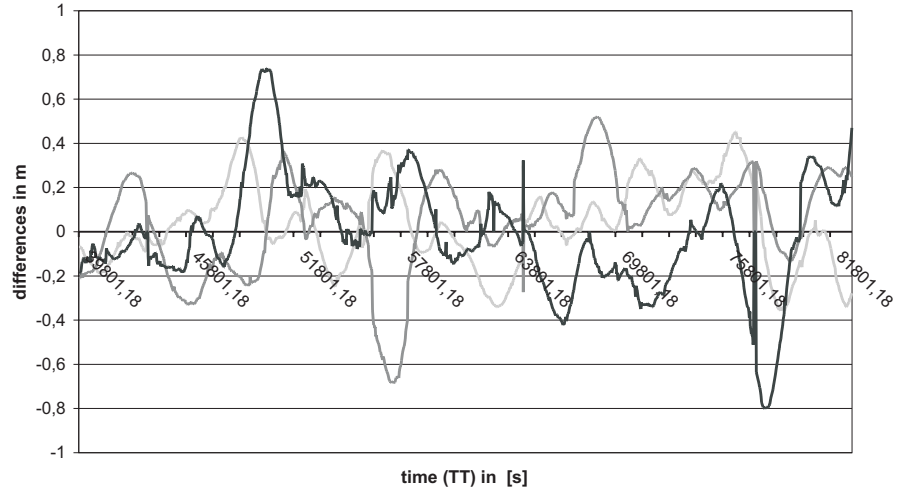

a)

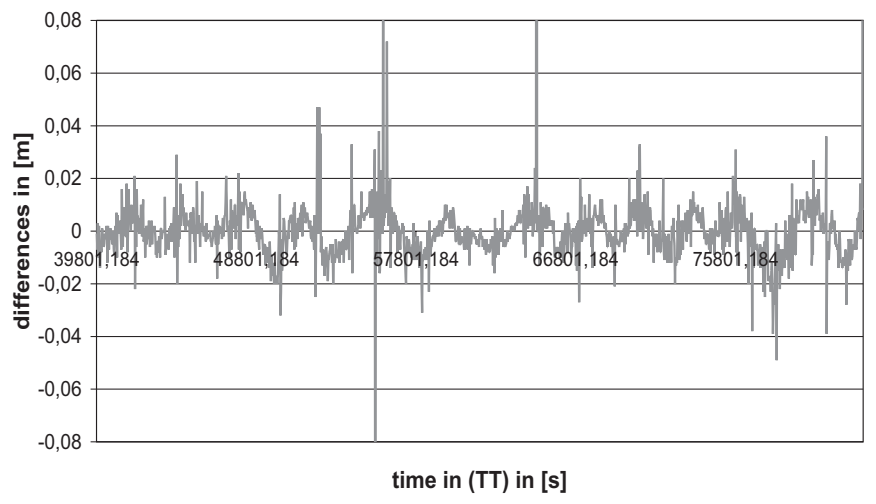

b)

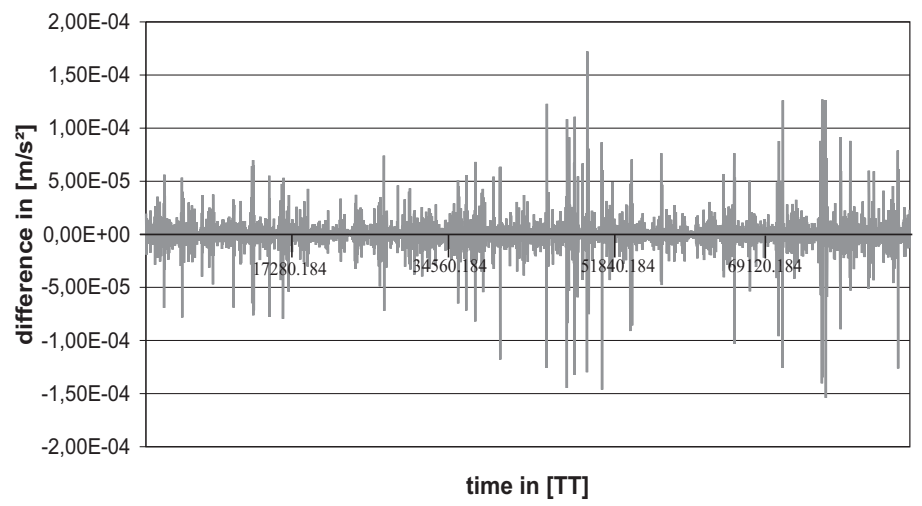

c)
Fig. 5. (a) difference of absolute ephemeris, (b) difference of baselines ( $x$-coordinate) and (c) difference of accelerations ( $x$-coordinate) between CHAMP RSO and kinematic orbit (day $141 / 2001$ ) in the qIRF. to the (smooth) Rapid Science (dynamic) orbit. Figures 5a to c illustrate the differences of (a) absolute coordinates, (b) baselines and (c) accelerations between kinematic and Rapid Science orbit.

The accuracy of the RSO is estimated by an independent comparison to Satellite Laser Ranging (SLR) measurements as $11 \mathrm{~cm}$ (Michalak et al., 2002) while the accuracy of the kinematic orbit can be classified in the range of $10-15 \mathrm{~cm}$ by a comparison to SLR (Svehla and Rothacher, 2002a, b). A second possibility to obtain information about the quality of the RSO is overlap analysis. From the $2 \mathrm{~h}$ overlap intervals of the $14 \mathrm{~h}$ RSO arcs we estimate an accuracy of the absolute RSO coordinates of $30 \mathrm{~cm}$ (Fig. 6a), while the accuracy of the RSO-baselines is $0.9 \mathrm{~cm}$ (Fig. 6b). Indeed, as the comparison to SLR illustrates, the accuracy of the RSO is much better, since the dynamic orbits are less accurate at the overlap intervals due to oscillation effects at the beginning and at the end of an arc. Thus, the accuracy of the baselines is even higher than the estimated $0.9 \mathrm{~cm}$ which means that they provide ideal reference values for the evaluation of the kinematic orbit baselines. Figure 5a illustrates the differences between the kinematic orbit and the RSO, which are in the level of $22 \mathrm{~cm}$. Though it is difficult to use this difference to estimate the real error level of the kinematic orbit due to errors in the RSO, we are able to extract from Fig. 5a that the errors of the kinematic orbit are far from being white noise (due to 


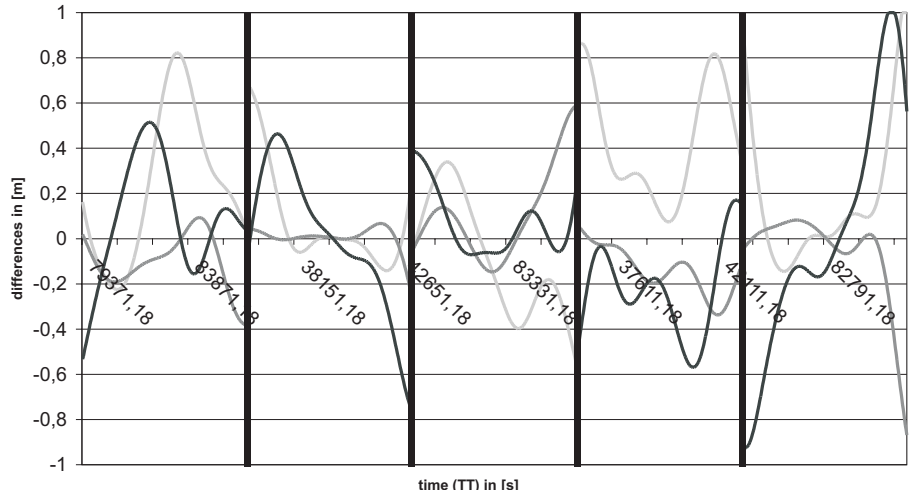

a)

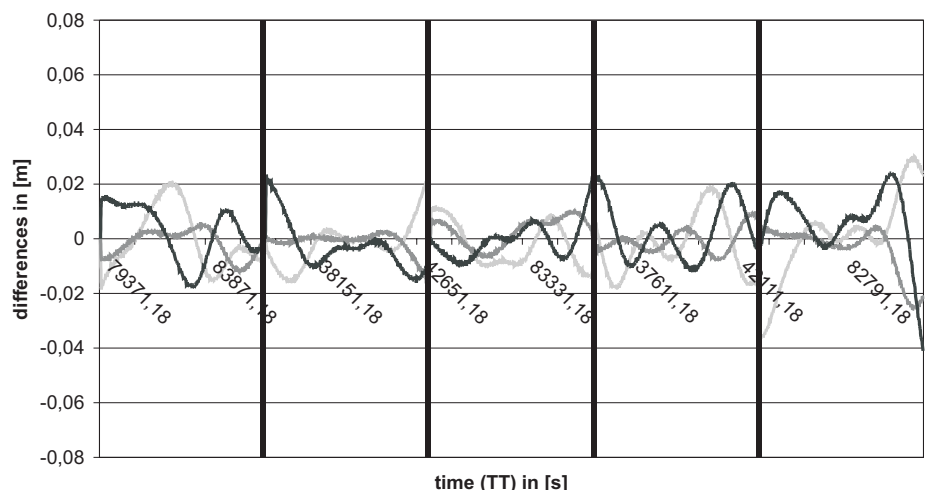

b)

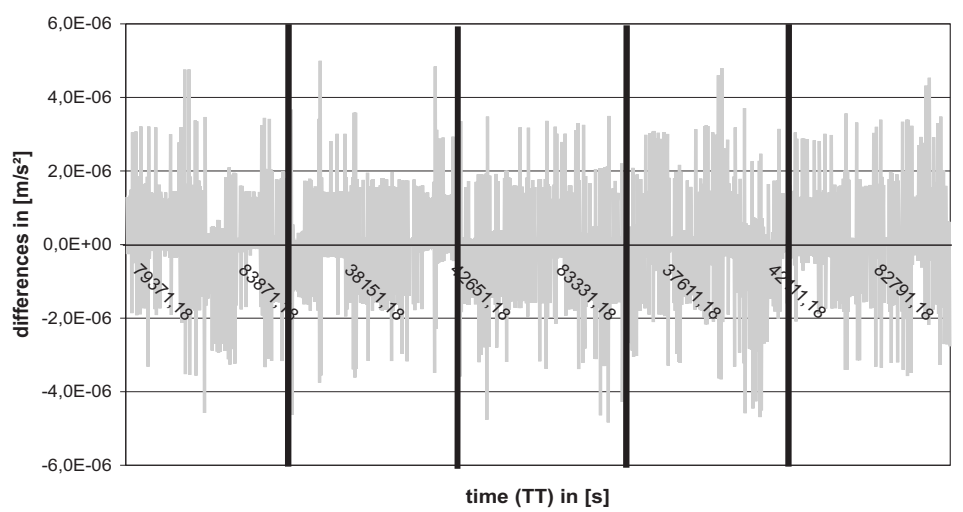

c)
Fig. 6. (a) differences of absolute RSO ephemeris, (b) RSO-baseline differences and (c) RSO acceleration differences as obtained from orbit overlaps of 6 arcs of CHAMP RSO (days 141$143 / 2001$ ) in the qIRF (5 overlap periods with 2 hours), $x$-, $y$-, $z$-coordinates (bright grey, dark grey, black). the smooth behaviour of the RSO). This becomes apparent in Fig. 5b where the differences of the baselines computed from the kinematic orbit and the RSO are plotted, which are in the level of $1.0 \mathrm{~cm}$ (if we disregard the few outliers) and which would have been in the $\mathrm{dm}-$ level for white noise.

Regarding the accuracy of the RSO baselines of at least $0.9 \mathrm{~cm}$ or better, the absolute accuracy of the kinematic orbit baselines lies within $1-2 \mathrm{~cm}$. Taking a value for the baseline - accuracy of $1.5 \mathrm{~cm}$ and the absolute kinematic orbit error (by the comparison to SLR) of $10 \mathrm{~cm}$, we obtain from their ratio $\sigma_{\Delta X_{i}} / \sigma_{X_{i}}=\sqrt{2} \sqrt{1-\rho}$ (resulting from error propagation) a correlation coefficient of about $\rho=0.99$, which we have applied in our simulations. From the $R M S$ of the difference of the accelerations between RSO and kinematic orbit of $1.9997 \mathrm{~m} / \mathrm{s}^{2}$ and the accuracy of RSO accelerations of $1.7 \cdot 10^{-6} \mathrm{~m} / \mathrm{s}^{2}$ estimated from overlaps we determine the accuracy of kinematic orbit accelerations in the level of $2 \mathrm{mGal}$, as obtained from simulations of Sect. 4.2. This should lead (Reubelt et al., 2002) to a geoid accuracy of at least $1 \mathrm{dm}$ for the complete CHAMP mission, which is comparable to present day long-wavelength geoid models, and demonstrates in general the applicability of the proposed method. Further refinement of the method will eventually lead to a contribution in improving these geoid models.

For the sake of completeness, we present the statistics of the analyzed 11-days kinematic orbit: 


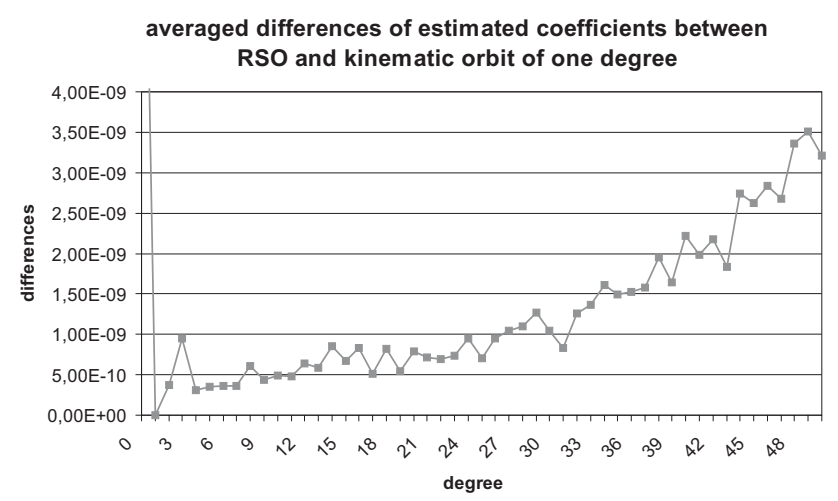

Fig. 7. Averaged differences of estimated spherical harmonic coefficients between kinematic and Rapid Science CHAMP orbit.

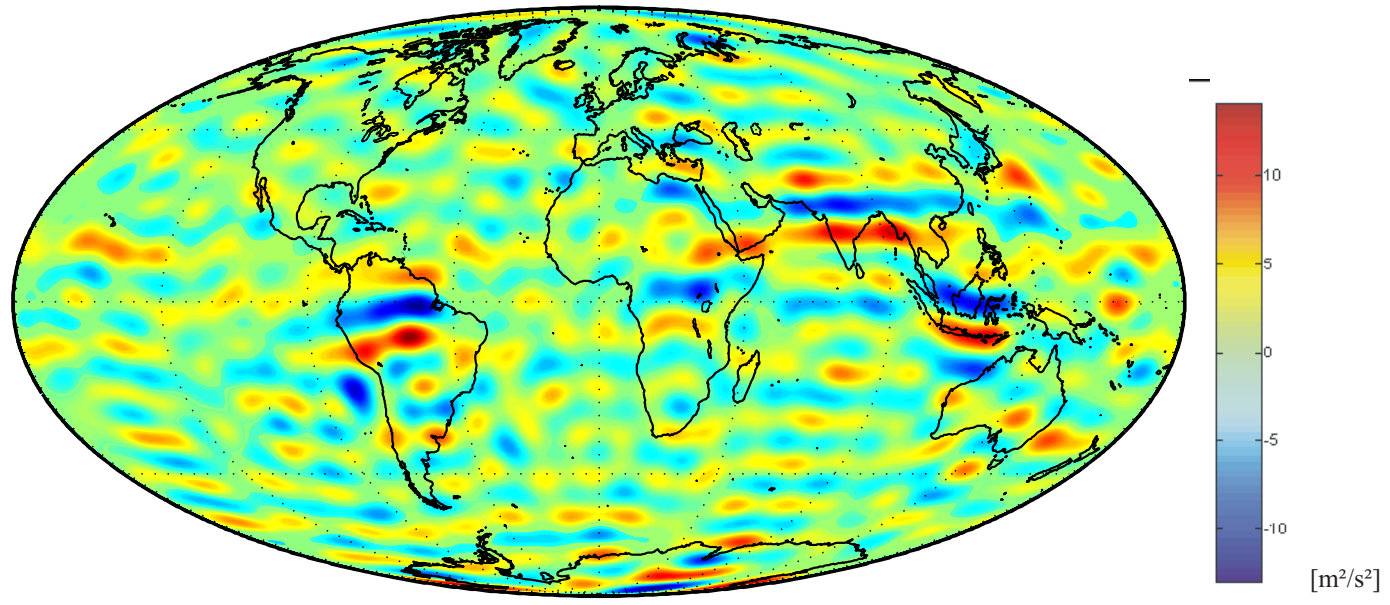

Fig. 8. Difference between recovered geopotential from a 11-days CHAMP-kinematic orbit and GRIM5_C1 up to degree/order 30/30 on the surface of a reference Earth with $R=6371 \mathrm{~km}$; without $c_{20}$ term; $R M S_{P o t}=3.2 \mathrm{~m}^{2} / \mathrm{s}^{2}$.

- number of analyzed coordinate-triples: 27360 ; number of data-gaps: 635 ;

- number of determined acceleration-triples ${ }^{1}: 25853$

- RMS of coordinates ${ }^{2} /$ baselines $^{2} /$ accelerations $^{2}$ : $0.22285 \mathrm{~m} / 0.01047 \mathrm{~m} / 1.9997 \cdot 10^{-5} \mathrm{~m} / \mathrm{s}^{2}$.

$\left({ }^{1}\right)$ : baselines with a deviation of $0.1 \mathrm{~m}$ in comparison to RSO have been neglected

$\left.{ }^{2}\right): R M S$ of differences between CHAMP RSO and CHAMP kinematic orbit

Figure 8 presents the difference between the recovered potential from the kinematic orbit and the GRIM5_C1 model up to degree/order 30/30. Obviously the estimated coefficients and the recovered potential from the kinematic orbit are noisier than those from the analysis of the Rapid Science orbit, but we have to notice that the kinematic orbit data set was shorter than the RSO data set. The highest differences seem to be in the regions of rough gravity field as for example the Andes or the Himalayas, but also in other areas bigger differences are visible. For the estimation up to degree/order
30/30 the recovered potential differs from GRIM5_C1 by $R M S_{P o t}=3.2 \mathrm{~m}^{2} / \mathrm{s}^{2}$, which corresponds to a $R M S$ of geoid differences of $\sim 30 \mathrm{~cm}$. If the kinematic orbit is analysed to a higher degree/order, for instance 50/50, the $R M S$ increases to $R M S_{P o t}=9.5 \mathrm{~m}^{2} / \mathrm{s}^{2}$, which means a $R M S$ of geoid differences of almost $1 \mathrm{~m}$. This is stated by Fig. 7, where we can extract the errors of the coefficients to degree 30 in the level of $1 \cdot 10^{-10}-1 \cdot 10^{-9}$, while they are increasing for higher degrees. We have to keep in mind, that only a 11-days kinematic CHAMP arc has been analysed. The investigation of longer and more accurate arcs will lead to a higher accuracy, even for the coefficients of higher degree.

\section{Conclusions}

First results from preliminary real CHAMP orbits by means a gravity field determination approach based on determined accelerations from a LEO satellite orbit are presented in this contribution to demonstrate arising problems with the method and the achievable accuracy. A numerical differentiation scheme is applied to determine the accelerations. To 
minimize noise amplification due to differentiation, baselines instead of absolute ephemeris are introduced, which can be determined more accurate since kinematic orbits are highly correlated. The RMS of the accelerations lies within 1$2 \mathrm{mGal}$, which leads to a long-wavelength geoid accuracy in the range of $20 \mathrm{~cm}$ from 1-week-arcs, as estimated from simulations in previous papers. Thus from the whole CHAMPmission, a long- wavelength geoid accuracy of at least $1 \mathrm{dm}$ can be expected. The analysis of a preliminary 10-days kinematic CHAMP orbit leads to a lower accuracy of $30 \mathrm{~cm}$. Since an improvement of kinematic orbits is expected in the near future (Svehla and Rothacher, 2002b), from the whole CHAMP mission also a geoid accuracy of at least $1 \mathrm{dm}$ is expected, which is at least a confirmation of state of the art models. But an improvement of existing gravity field models seems at the moment hard to realize. A comparison between the proposed method and existing approaches has to be done to demonstrate if our algorithm can compete, in particular if and which parts of the gravitational field can be determined better. Future research should focus mainly on noise reduction in the process of acceleration determination, for instance by means of smoothing interpolation functions or regression methods, since the accuracy level of $1-2 \mathrm{mGal}$ still seems to high.

\section{References}

Austen, G. and Reubelt, T.: Räumliche Schwerefeldanalyse aus semi-kontinuierlichen Ephemeriden niedrigfliegender GPSvermessener Satelliten vom Typ CHAMP, GRACE und GOCE, M. Sc. Thesis, Geodetic Institute, University of Stuttgart, Germany, 2000.

Austen, G., Grafarend, E. W., and Reubelt, T.: Analysis of the Earth's Gravitational Field from Semi-Continuous Ephemeris of a Low Earth Orbiting GPS-Tracked Satellite of Type CHAMP, GRACE or GOCE, in: International Association of Geodesy Symposia, Vol. 125, Vistas for Geodesy in the New Millennium, (Eds) Adam, J. and Schwarz, K. P., Axel Springer Verlag, 2002.

Belikov, M. V. and Taybatorov, K. A.: An efficient algorithm for computing the Earth's gravitational potential and its derivatives at satellite altitudes, Manuscr. Geod. 17, 104-116, 1992.

Biancale, R., Balmino, G., Lemoine, J. M., Marty, J. C., Moyno, B., Barlier, F., Exertier, P., Laurain, O., Gegout, P., Schwintzer, P., Reigber, Ch., Bode, A., Gruber, Th., König, R., Massmann, F. H., Raimondo, J. C., Schmidt, R., and Zhu, S. Y.: A new global Earth's gravity field model from satellite orbit perturbations: GRIM5-S1, Geophys. Res. Lett., 27, 3611-3614, 2000.

Engeln-Müllges, G. and Reutter, F.: Numerische Mathematik für Ingenieure (Numerical mathematics for engineers), BI Wissenschaftsverlag, Mannheim-Wien-Zürich, 1988.

Grafarend, E. and Vancek, P.: On the weight estimation in leveling, National Oceanic and Atmospheric Adminstration (NOAA), Report NOS 86, NGS 17, Rockville, 1980.

Gruber, T., Bode, A., Reigber, Ch., Schwintzer, P., Balmino, G., Bancale, R., and Lemoine, J. M.: GRIM5-C1: combination solution of the global gravity field to degree and order 120, Geophys. Res. Lett., 27, 4005-4008, 2000.

Hartmann, T. and Wenzel, H. G.: The HW95 tidal potential catalogue, Geophys. Res. Lett., 22, 24, 3553-3553, 1995.

King-Hele, D.: Satellite orbits in an atmosphere: theory and applications, Blackie Ltd, Glasgow, London, UK, 1987.

Koop, R. and Stelpstra, D.: On the computation of the gravitational potential and its first and second order derivatives, Manuscr. Geod. 14, 373-383, 1989.

Lemoine, F. G., Kenyon, S. C., Factor, J. K., Trimmer, R. G., Pavlis, N. K., Chinn, D. S., Cox, C. M., Klosko, S. M., Luthcke, S. B., Torrence, M. H., Wang, Y. M., Williamson, R. G., Pavlis, E. C., Rapp, R. H., and Olson, T. R.: The NASA GSFC and NIMA Joint Geopotential Model, NASA Goddard Space Flight Center, NASA/TP-1998-206861, Greenbelt, Maryland, USA, 1998.

McCarthy, D. D.: IERS Conventions 1996, IERS Technical Note 21, Observatoire de Paris, Paris, France, 1996.

Michalak, G., Baustert, G., Koenig, R., and Reigber, Ch.: CHAMP Rapid Science Orbit Determination - Status and Future Prospects, in: First CHAMP Mission Results for Gravity, Magnetic and Atmospheric Studies, (Eds) Reigber, c., Lühr, H., and Schwintzer, P., 98-103, Axel Springer-Verlag, 2003.

Reubelt, T., Austen, G., and Grafarend, E. W.: Harmonic analysis of the Earth's gravitational field by means of semi-continuous ephemeris of a Low Earth Orbiting GPS tracked satellite - case study: CHAMP, J. Geodesy, in press, 2003.

Svehla, D. and Rothacher, M.: Kinematic Orbit Determination of LEOs Based on Zero or Double-difference Algorithms Using Simulated and Real SST GPS Data, in: International Association of Geodesy Symposia, Vol. 125, Vistas for Geodesy in the New Millennium, (Eds) Adam, J. and Schwarz, K. P., Axel Springer Verlag, 2002a.

Svehla, D. and Rothacher, M.: Kinematic and reduced-dynamic precise orbit determination of low earth orbiters, Adv. Geosciences, 1, 47-56, 2003.

Wahr, J.: Earth tides, Global Earth physics, AGU Reference Shelf 1: 40-45, 1995. 